\title{
Omitting Preoperative Lymphoscintigraphy Does Not Decrease Sentinel Lymph Node Detection Rate in Vulvar Cancer
}

\author{
Kasey Marie Roberts", Aine Emma Clements, John Ottis Elliott, Kellie Susan Rath, \\ Gary Craig Reid
}

Department of Obstetrics and Gynecology, Riverside Methodist Hospital, Columbus, USA

Email address:

Kasey.m.roberts@gmail.com(K. M. Roberts), Aine.clements@ohiohealth.com(A. E. Clements), John.elliott@ohiohealth.com(J. O. Elliott), Kellie.rath@ohiohealth.com(K. S. Rath), Gary.reid@ohiohealth.com(G. C. Reid)

${ }^{*}$ Corresponding author

\section{To cite this article:}

Kasey Marie Roberts, Aine Emma Clements, John Ottis Elliot, Kellie Susan Rath, Gary Craig Reid. Omitting Preoperative Lymphoscintigraphy Does Not Decrease Sentinel Lymph Node Detection Rate in Vulvar Cancer. International Journal of Clinical Oncology and Cancer Research. Vol. 3, No. 3, 2018, pp. 30-34. doi: 10.11648/j.ijcocr.20180303.13

Received: June 19, 2018; Accepted: July 16, 2018; Published: August 9, 2018

\begin{abstract}
Sentinel lymph nodes in vulvar cancer can be detected in a variety of ways. A method described in GOG-173 involves injection of technetium- $99 \mathrm{~m}$ and isosulfan blue intraoperatively. Lymphoscintigraphy, for which the technetium- $99 \mathrm{~m}$ is injected preoperatively, became required two years into the study due to unpublished evidence that lymphoscintigraphy improved intraoperative sentinel lymph node localization. Despite this amendment, the need for preoperative lymphoscintigraphy has been questioned. The primary objective of this study was to determine the detection rate of sentinel lymph nodes in vulvar cancer when preoperative lymphoscintigraphy is omitted. The secondary objective was to compare the sentinel lymph node detection rate when lymphoscintigraphy is omitted to the rate reported in GOG-173, in which preoperative lymphoscintigraphy was performed. The tertiary objective was to determine lymphoscintigraphy cost at one institution. Patients with vulvar cancer who underwent sentinel lymph node dissection at a single institution from 2008 to 2016 were identified. All but one patient had intraoperative peritumoral injection of both technetium- $99 \mathrm{~m}$ and patent blue dye. Patients were excluded if preoperative lymphoscintigraphy was performed. Information on demographics, pathology, and outcomes were collected. Descriptive statistics were used for patient demographics, tumor characteristics, and the detection rate. A 1sample proportion test was used to compare our detection rate to data available from GOG-173. Percentages were rounded to the nearest whole number. Current procedural terminology codes were used to estimate lymphoscintigraphy cost. Fifty patients were identified and 32 patients were deemed eligible for the study. The sentinel lymph node detection rate was $97 \%$ per patient, which was not statistically different from the rate of $92 \%$ reported in GOG-173 $(p=0.347)$. It was determined that lymphoscintigraphy cost $\$ 5,288.22$ per imaging study. In this study, omitting preoperative lymphoscintigraphy did not decrease sentinel lymph node detection rates when compared to GOG-173. Furthermore, there is a substantial cost associated with lymphoscintigraphy, although this cost may vary between institutions.
\end{abstract}

Keywords: Lymphoscintigraphy, Sentinel Lymph Node, Vulvar Cancer

\section{Introduction}

Vulvar cancer is the fourth most common gynecologic malignancy with 6,020 new cases and 1,150 deaths attributable to the disease projected to occur in 2017 [1]. Lymphatic metastases are one of the most important prognostic variables in this disease; survival rates decrease by approximately $50 \%$ when nodal metastases are present [2]. Fortunately, only $20-30 \%$ of vulvar cancers demonstrate nodal metastases at the time of diagnosis [3, 4]. When vulvar cancer does spread to lymph nodes (LNs), it most commonly metastasizes to the inguinofemoral LNs, but can also metastasize much less commonly to the pelvic LNs [5].

Unfortunately, full inguinofemoral lymphadenectomy 
(IFLND) is associated with complications such as lymphedema, wound break down, and operative site infection in $20-70 \%$ of cases [6]. Because of the high incidence of complications with IFLND, sentinel lymph node dissection (SLND), which has a complication rate of only $1.9-11.7 \%$, began to be studied as a replacement for IFLND in 1994 [7, 8]. One of the prominent study protocols on the safety of SLND in evaluating LN metastases in vulvar cancer is known as GOG-173. GOG-173 was a large surgical feasibility study performed on patients with squamous cell carcinoma of the vulva that measured $\geq 2 \mathrm{~cm}$ and $\leq 6 \mathrm{~cm}$. Enrolled patients had sentinel lymph nodes (SLN) identified, excised, and then a full inguinal lymphadenectomy performed for analysis. For tumors less than $4 \mathrm{~cm}$, the study demonstrated a false negative predictive value of only $2.0 \%$ with SLND, supporting SLND as a new method for detecting LN metastases in vulvar cancer [6].

SLN can be detected in a variety of ways. The method described in GOG-173 involves injection of technetium-99m and isosulfan blue intraoperatively. Lymphoscintigraphy (LSG), for which the technetium-99m is injected preoperatively, became required two years into the study due to unpublished evidence that LSG improved intraoperative SLN localization [6]. Despite this amendment, the need for preoperative LSG has been questioned. Although proponents argue that it increases diagnostic accuracy while reducing morbidity $[3,9,10]$, opponents point out that LSG adds cost, time, and patient discomfort [11, 12]. Indeed, GOG-173 study accrual was noted to decrease following its amendment to require LSG, highlighting the sensitivity of patients and institutions to the disadvantages of LSG. Furthermore, omitting LSG may not significantly affect SLN detection rate. At our institution, we routinely omit preoperative LSG given its disadvantages and unclear benefit.

The primary objective of this study was to determine the detection rate of SLN in vulvar cancer when preoperative LSG is omitted. The secondary objective was to compare the SLN detection rate when LSG is omitted to the rate reported in GOG173, in which preoperative LSG was performed. The tertiary objective was to determine LSG cost at one institution.

\section{Method}

The Riverside Methodist Hospital (RMH) Institutional Review Board approved this study (IRB \#OH1-16-00668). This is a retrospective study that was done by chart review. Patients identified had been diagnosed with vulvar cancer at our institution from 2008 to 2016. A chart review was then performed and those patients who had undergone SLND were included. Patients were excluded if preoperative LSG had been performed.

Demographic data, including age, race, history of smoking and known history of vulvar dermatoses, was collected. Information on tumor characteristics was also collected, including tumor histology, laterality, size, grade, depth of invasion, lymphovascular space invasion, and stage of cancer. Most of the tumor characteristic information was obtained from pathology reports; in situations in which these were unavailable or lacking necessary information, office descriptions of the tumors were utilized. Using operative and pathology reports, details of SLNs were collected, including number collected, method of detection (hot, blue, or hot and blue) and the number of positive or negative SLNs. Operative reports were used to determine if IFLND was performed, and if performed, the indication, laterality, and number of nodes collected for each IFLND. Postoperative information collected included time to first postoperative follow up visit, date of last documented office visit, and postoperative complications (including wound infection, wound breakdown, lymphocyst formation, and lymphedema) and their interval from surgery. Data was also collected on the number of patients with a cancer recurrence and the number of patients who died during the study period. Finally, although no included patients had preoperative LSG, there were several who had preoperative CT. It was noted which patients had preoperative CT performed, and when this preoperative imaging identified positive LNs.

Descriptive statistics were used to determine patient demographics, tumor characteristics, and SLN detection rate. A 1-sample proportion test was used to compare our detection rate to that reported in GOG-173 $\mathrm{z}_{0}=\left(\mathrm{p}-\mathrm{hat}-\mathrm{p}_{0}\right) / \mathrm{V}$ $\left(\left(\mathrm{p}_{0}\left(1-\mathrm{p}_{0}\right) / \mathrm{n}\right)\right)$. Ninety-five percent confidence intervals were used to descriptively compare our variables pertaining to SLND to those same variables reported in GOG-173. These variables included percent of SLN detected on per patient and per groin bases, percent of SLN positive for metastases, and method of SLN detection. All percentages were rounded to the nearest whole number. Finally, we determined the cost of LSG at our institution by providing our cost center with the current procedural terminology (CPT) codes associated with LSG.

Of note, our institution performs SLND in a slightly different manner than most institutions. Generally, there are both preoperative and intraoperative components to a SLND. First, the patient presents for a preoperative LSG, at which time technetium- $99 \mathrm{~m}$ is injected peritumorally and the LSG is performed. Next, the patient is taken to the OR where intraoperative peritumoral injection of patent blue dye is performed. Finally, the SLNs are identified via a combination of a nanoprobe for detection of the technetium-99m and direct visualization of the patent blue dye. Our institution's protocol deviates from the above as preoperative LSG is typically not performed. Instead, the surgeon requests the technetium-99m via a fax order the day before surgery. On the day of surgery, a technician brings the technetium- $99 \mathrm{~m}$ to the operating room in a protective case. Just after the patient has undergone induction of general anesthesia, the surgeon injects both the technetium-99m and the patent blue dye peritumorally. The used technetium- $99 \mathrm{~m}$ syringes then go back in their case, and the OR staff returns the materials back to the nuclear medicine staff. The patient is then prepped and draped in the usual sterile fashion and the procedure is begun. Finally, the SLNs are identified via use of the nanoprobe and direct visualization as described above. It is 
therefore important to note that all of our patients receive injections of both technetium-99m and patent blue dye.

Another notable difference between our institution's SLND and those performed at other institutions includes eligibility criteria for SLND. Generally, tumors that are considered to be eligible for SLND have diameters less than four centimeters, depths of invasion greater than one millimeter, and are unifocal. Additionally, any tumor associated with palpable groin LNs are typically excluded. Given that these conditions are still evolving, our institution has occasionally deviated from the suggested SLND criteria in situations in which it was felt that a SLND would be the best management option for the patient.

\section{Result}

We identified 50 patients with vulvar cancer at our institution between 2008 and 2016. We excluded 16 patients because they had had preoperative LSG performed and two patients because they did not have enough information to be included, leaving 32 patients available for evaluation. Demographic information on the included patients is available in Table 1. In general, this was a largely Caucasian population that was postmenopausal (average age of 67.7 years at the time of their vulvar cancer diagnosis). Most were either current or former smokers and a significant number reported a history of vulvar dermatoses. Ninety-one percent of the tumors were squamous cell carcinomas. The mean tumor size was $1.8 \mathrm{~cm}$ and the mean depth of invasion was $3.2 \mathrm{~mm}$. Sixty-one percent were stage IB on final pathology.

Table 1. Patient demographics.

\begin{tabular}{lll}
\hline Demographic & Number of Patients & \% \\
\hline Age (years) & 6 & \\
$\leq 50$ & 17 & 19 \\
$50-79$ & 9 & 53 \\
$>80$ & & 28 \\
Race & 27 & \\
White & 3 & 84 \\
Black & 2 & 9 \\
Unknown/not specified & & 6 \\
& & \\
Smoker & 6 & 19 \\
Current & 11 & 34 \\
History & 15 & 47 \\
Never/no known history & & \\
& & \\
Vulvar Dermatoses & 11 & 34 \\
Ever in life & 21 & 66 \\
Never/no known history & & \\
\hline
\end{tabular}

Table 2 outlines the tumor characteristics.

Table 2. Tumor characteristics.

\begin{tabular}{lll}
\hline Characteristic & Number of Patients & \% \\
\hline Histology & & \\
Squamous cell carcinoma & 29 & 91 \\
Melanoma & 2 & 6 \\
Adenocarcinoma & 1 & 3 \\
\hline
\end{tabular}

\begin{tabular}{|c|c|c|}
\hline Characteristic & Number of Patients & $\%$ \\
\hline \multicolumn{3}{|l|}{ Laterality } \\
\hline Unilateral & 11 & 34 \\
\hline Midline & 20 & 63 \\
\hline Unknown/not specified & 1 & 3 \\
\hline \multicolumn{3}{|l|}{ Tumor size (cm) } \\
\hline$<2$ & 16 & 50 \\
\hline $2.0-2.9$ & 6 & 19 \\
\hline $3.0-3.9$ & 5 & 16 \\
\hline $4.0-4.9$ & 2 & 6 \\
\hline$\geq 5.0$ & 2 & 6 \\
\hline Unknown/not specified & $1 *$ & 3 \\
\hline \multicolumn{3}{|l|}{ Tumor grade } \\
\hline Well-differentiated & 13 & 41 \\
\hline Moderately-differentiated & 10 & 31 \\
\hline Poorly-differentiated & 3 & 9 \\
\hline Unknown/not specified & 6 & 19 \\
\hline \multicolumn{3}{|l|}{ Depth of invasion (mm) } \\
\hline$\leq 1$ & 2 & 6 \\
\hline $1-5$ & 21 & 66 \\
\hline$\geq 5$ & 6 & 19 \\
\hline Unknown/not specified & $3 * *$ & 9 \\
\hline \multicolumn{3}{|l|}{ LVSI } \\
\hline Present & 2 & 6 \\
\hline Absent & 23 & 72 \\
\hline Unknown/not specified & 7 & 22 \\
\hline \multicolumn{3}{|l|}{ Tumor stage } \\
\hline Stage I & 23 & 72 \\
\hline Stage II & 1 & 3 \\
\hline Stage III & 7 & 22 \\
\hline Stage IV & 0 & 0 \\
\hline Unknown & 1 & 3 \\
\hline
\end{tabular}

LVSI: Lymphovascular space invasion

*Lesion had been excised before being evaluated by GYN/ONC at RMH. **SLND was performed, but lesion itself was not excised due to size.

For all patients except one (in which only patent blue dye was used), both technetium-99m and patent blue dye were used for the SLND. SLNs were detected in $31 / 32$ (97\%) patients, and in 48/49 (98\%) groins. Of note, in the one patient in whom a SLN could not be detected, only patent blue dye was used as the technetium-99m was not available. The median number of SLNs identified per person was two and-a-half (range $1-27$ ), and per groin was two (range $0-$ 6) on the left and one (range $1-23$ ) on the right. SLN detection methods were available for all but six SLN; two operative reports did not comment on whether or not the SLNs were hot, blue, or both.

Of the 114 SLNs that were removed, four were noted to be positive $(4 \%)$, which corresponded to a $13 \%$ positive SLN rate on a per patient basis. Of those women who did have positive SLNs intraoperatively, all were found to be positive when examined during final pathologic review. One woman who had a negative intraoperative SLN was found to have a positive SLN on final pathologic review. This patient was offered radiation but she declined. She continues to live, approximately one year since her surgery, without a recurrence. One patient's operative report was not available 
to verify that the intraoperative pathology was the same as the final pathology. Table 3 compares our SLN detection rate with the rate described in GOG-173 and provides 95\% confidence intervals for qualitative comparisons between the
SLN detection methods in the two studies. Via a 1-sample proportion test, we found a non-statistically significant difference between our detection rate and that reported in GOG-173.

Table 3. Comparison of SLN data: Roberts et al. vs. GOG-173.

\begin{tabular}{lll}
\hline Variable & Roberts et al. & GOG-173 \\
\hline \multirow{2}{*}{ SLN detected per patient } & $31 / 32(97 \%)$ & $418 / 452(92 \%)$ \\
& $95 \%$ CI: $84.3-99.5$ & $95 \%$ CI: $89.7-94.6$ \\
SLN detected per groin & $48 / 49(98 \%)$ & $593 / 772(77 \%)$ \\
& $95 \%$ CI: $89.3-99.6$ & $95 \%$ CI: $73.7-79.7$ \\
Positive SLN rate per patient & $4 / 32(13 \%)$ & $132 / 418(32 \%)$ \\
& $95 \%$ CI: $5.0-28.1$ & $95 \%$ CI: $37.6-56.7$ \\
SLN detected as both hot and blue & $101 / 114(89 \%)$ & $254 / 418(61 \%)$ \\
& $95 \%$ CI: $81.5-93.2$ & $95 \%$ CI: $56.0-65.3$ \\
SLN detected as hot only & $5 / 114(4 \%)$ & $64 / 418(15 \%)$ \\
& $95 \%$ CI: $1.9-9.9$ & $95 \%$ CI: $12.2-19.1$ \\
SLN detected as blue only & $2 / 114(2 \%)$ & $100 / 418(24 \%)$ \\
Unknown & $95 \%$ CI: $0.5-0.6$ & $95 \%$ CI: $20.1-28.2$ \\
\hline
\end{tabular}

SLN: Sentinel lymph node

GOG-173: Gynecologic Oncology Group - 173

95\% CI: 95\% Confidence Interval

IFLND was performed in $28 \%$ patients, but only one of these cases was secondary to not being able to identify SLN. In the other cases, IFLND was performed for the following reasons: concern that the SLN was not the true SLN (one out of nine), palpable LN (two out of nine), previous groin dissection (one out of nine), and positive intraoperative SLN (three out of nine). One operative report did not specify why IFLND was performed.

Although none of the patients evaluated in our study had preoperative LSG, most (66\%) of them did have preoperative PET/CT performed, most of which were performed two to four weeks prior to SLND. Of the seven PET/CTs that detected metabolically active LNs, none of the corresponding SLND returned with any positive LNs. Furthermore, three of the $14(21 \%)$ PET/CTs that did not detect any metabolically active LNs did have nodal disease on final pathology.

Ninety-seven percent of patients were seen for postoperative evaluation; the one patient who was not seen had requested that she follow up with her primary gynecologist secondary to travel issues. Ninety percent of these patients were seen within one month of their surgery. After dividing the patients into those who only underwent SLND $(n=23)$ from those who underwent SLND and IFLND $(\mathrm{n}=9)$, complication rates were evaluated. Respective complication rates for SLND vs. SLND + IFLND were as such: wound infection: $35 \%$ vs. 56\%; wound breakdown: $9 \%$ vs. 33\%; lymphocyst formation: $9 \%$ vs. $11 \%$; and lymphedema: $4.3 \%$ vs. $33 \%$. Eighty percent of these complications were noted within one month of surgery.

Patients were followed for a median of 21 (range 1 - 106) months. At a median of 21.8 (range 3.3 - 71.3) months from their surgery, $22 \%$ of patients had a recurrence of their cancer. By the end of the follow-up period, $13 \%$ of patients had died at a median of $32.1(21.3$ - 39.9) months from their surgery. Of those patients who had died, all had suffered a cancer recurrence, although only two were known to die from their cancer, in particular.

Finally, LSG cost was determined by providing our institution's cost center the CPT codes used for LSG. We obtained the price of the imaging study $(\$ 5,044.22$, CPT $38792)$,) and the radiologist reading the image (\$244.00, no associated CPT code used). Therefore, LSG costs $\$ 5,288.22$ per patient at our institution.

\section{Discussion}

The primary objective of this study was to determine the SLN detection rate amongst patients with vulvar cancer who did not undergo preoperative LSG. Peritumoral injection of technetium-99m and patent blue dye were injected at the time of surgery for all but one of the patients studied; due to a supply issue, one patient was only injected with patent blue dye. A 26-study meta-analysis from the NIH published in 2013 reported the overall SLN detection rate using both technetium-99m and patent blue dye as 97.7\% [13]. This finding is corroborated by a 49-study 2013 meta-analysis which also reported a per patient SLN detection rate of $94.4 \%$ [8]. Subjectively, the SLN detection rates in GOG-173 $(92.5 \%)$ and our study (97\%) are similar to the above reports. Moreover, when our rate was statistically compared to GOG173 , the detection rates were not found to be statistically significantly different $(\mathrm{p}=0.347)$.

An article published in 2010 in the European Journal of Obstetrics \& Gynecology and Reproductive Biology sought to address the same question as our study [10]. They reported a SLN detection rate of $98 \%$ with use of preoperative LSG compared to $94 \%$ without it and concluded that preoperative LSG does improve SLN detection rates. However, only patent blue dye was used in the group without LSG in this study, a factor that could account for the lower detection rate. Furthermore, even with the patent blue dye alone, the difference that they found was not reported to be statistically significant. In 
our study, there was only one patient in which SLNs were unable to be identified. Similar to the above study, this one patient only had patent blue dye injected at the time of surgery.

The utility of preoperative imaging has been called into question given its frequent inaccuracy. For instance, it has been reported that the majority of patients with preoperative LSG SLN non-visualization will have at least one SLN detected intraoperatively [3]. Furthermore, the utility of LSG in lymphatic mapping for midline lesions is known to be limited [10, 13]. As an example, in GOG-173 32 (30\%) women with tumors invading or crossing the midline had unilateral drainage on LSG, yet four of these $32(12.5 \%)$ women had metastatic disease in the contralateral groin [6]. In our study, $65 \%$ of patients underwent preoperative $\mathrm{PET} / \mathrm{CT}$. With a positive predictive value of 0 and a negative predictive value of 0.79 , we found our PET/CTs to be unreliable. These findings further support the notion that imaging prior to SLND for vulvar cancer, even with PET/CT, may not increase the SLN detection rate.

A cost analysis of LSG at our institution determined that each LSG costs $\$ 5,288.22$. Therefore, at our institution, we estimate that by omitting LSG for the 32 patients over the eight-year study period, $\$ 169,223.04$ health care dollars were saved. The magnitude of LSG cost may vary between healthcare organizations.

Our study has several limitations. Most importantly, it is a retrospective observational study with a small sample size and no internal control. Additionally, we are drawing a comparison between two very different study populations, given that our study population is a retrospectively studied, small group, and the GOG-173 study population is a prospectively selected, very large group. Comparing these two groups is not necessarily statistically viable. Yet, while we understand that the two groups cannot be compared headto-head, we feel that this finding provides at least a preliminary understanding that omitting LSG may not affect SLN detection rate. Additional research is certainly required before this information can be utilized in clinical practice.

Future considerations consist of performing a prospective study with an internal control, as opposed to comparing results to an outside study as this study has. Additionally, long-term studies on how omission of preoperative LSG affects recurrence and/or survival rates in vulvar cancer would be of value. Finally, from a cost-savings perspective, an analysis should be performed that addresses whether operating room time, and therefore healthcare dollars, are saved when a preoperative "LN map" is available (via LSG).

\section{Conclusion}

Based on the results of this study, when both patent blue dye and technetium-99m are injected intraoperatively, omitting preoperative LSG does not decrease the detection rate of SLN. Additionally, preoperative imaging is not necessarily accurate at identifying positive SLN. Finally, LSG omission provides benefits from a financial perspective.

\section{References}

[1] American Cancer Society [Internet]. Atlanta (GA): American Cancer Society, 2017. Cancer Facts \& Figures [updated 2017; cited 2017 Jun 08]. Available from:

https://www.cancer.org/cancer/vulvar-cancer/about/keystatistics.html.

[2] Oonk MH, van Hemel BM, Hollema H, de Hullu JA, Ansink AC, Vergote I, et al. Size of sentinel-node involvement and survival in early stage vulvar cancer: results from GROINSS-V, a multicenter observational study. Lancet Oncol. $2010 \mathrm{Jul}$; 11 (7):646-52.

[3] Giammarile F, Bozkurt MF, Cibula D, Pahisa J, Oyen WJ, Paredes $\mathrm{P}$, et al. The EANM clinical and technical guidelines for lymphoscintigraphy and sentinel node localization in gynaecological cancers. Eur J Nucl Med Mol Imaging. 2014 Jul; 41 (7):1463-77.

[4] Gonzale Bosquet J, Kinney W, Russell A, Gaffey T, Magrina J, Podratz K. Risk of Occult Inguinofemoral Lymph Node Metastasis From Squamous Carcinoma of the Vulva. Int J Radiat Oncol Biol Phys. 2003 Oct 1; 57 (2):419-24.

[5] Hacker H, Eifel P, van der Velden J. Cancer of the vulva. Int J Gynaecol Obstet. 2015 Sept 2; 131:S76-S83.

[6] Levenback CF, Ali S, Coleman RL, Gold MA, Fowler JM, Judson PL. Lymphatic Mapping and Sentinel Lymph Node Biopsy in Women With Squamous Cell Carcinoma of the Vulva: A Gynecologic Oncology Group Study. J Clin Oncol. 2012 Nov 1; 30 (31):3786-91.

[7] Covens A, Vella E, Kennedy E, Reade CJ, Jimenez W, Le T. Sentinel lymph node biopsy in vulvar cancer: Systematic review, meta-analysis, and guideline recommendations. Gynecol Oncol. 2015 May; 137 (2):351-61.

[8] Hassanzade M, Attaran M, Treglia G, Yousefi Z, Sadeghi R. Lymphatic mapping and sentinel node biopsy in SCC of the vulva: Systematic review and meta-analysis of the literature. Gynecol Oncol. 2013 Jul; 130 (1):237-45.

[9] Hubalewska-Dydejczk A, Sowa-Staszczak A, Huszno B. Current application of sentinel lymph node lymphoscintigraphy to detect various cancer metastases. Hell J Nucl Med. 2006 Jan-Apr; 9 (1):5-9.

[10] Lindell G, Jonsson C, Ehrsson RJ, Jacobsson H, Danielsson KG, Källström $\mathrm{BN}$ et al. Evaluation of preoperative lymphoscintigraphy and sentinel node procedure in vulvar cancer. Eur J Obstet Gynecol Reprod Biol. 2010 Sep; 152 (1):91-5.

[11] McCann G, Cohn D, Jewell E, Havrilesky, LJ. Lymphatic mapping and sentinel lymph node dissection compared to complete lymphadenectomy in the management of early-stage vulvar cancer: A cost-utility analysis. Gynecol Oncol. 2015 Feb; 136 (2):300-4.

[12] Slomovitz B, Coleman R, Oonk M, van der Zee A, Levenback C. Update on sentinel lymph node biopsy for early-stage vulvar cancer. Gynecol Oncol. 2015 Aug; 138 (2):472-7.

[13] Meads C, Sutton A, Małysiak S, Kowalska M, Zapalska A, Rogozinska E, et al. Sentinel lymph node status in vulval cancer: systematic review of test accuracy and decisionanalytic model-based economic evaluation. Health Technol Assess. 2013 Dec; 17 (60):1-216. 